\title{
Gastric adenocarcinoma: clinicopathologic differences among Hispanics and non-Hispanic whites. A single Institution's experience over 14 years
}

\author{
Narjust Duma a , Larysa J. Sanchez ${ }^{a}$, Yulanka S. Castro a , Andrew A. Jennis ${ }^{b}$, Donald A. McCain ${ }^{\text {, }}$ \\ Martin E. Gutierrez ${ }^{\mathbf{b}}$, Zubin M. Bamboat ${ }^{\mathbf{b}}$ \\ Rutgers-New Jersey Medical School, Newark; John Theurer Cancer Center, Hackensack University Medical Center, \\ Hackensack, USA
}

\begin{abstract}
Background Gastric cancer is a leading cause of cancer death worldwide and has significant ethnic and socioeconomic differences in distribution. The aim of this study was to compare clinicopathologic characteristics and survival between Hispanics $(\mathrm{H})$ and non-Hispanic whites (NHW) with gastric cancer.

Methods We reviewed the records of all patients diagnosed with gastric cancer between 1999 and 2013 at our institution. A total of 638 patients were studied. Demographics, tumor characteristics and survival rate were analyzed. Kaplan-Meier was used for survival analysis.

Results There were $101 \mathrm{H}$ and 537 NHW. The median age at diagnosis was 63 years in $\mathrm{H}$ and 69 years in NHW. At diagnosis, 48 (48\%) of $\mathrm{H}$ patients had stage IV disease compared with $195(36 \%)$ of NHW $(\mathrm{P}<0.03)$. H were more likely to have distal cancers and poorly differentiated tumors compared to NHW ( $44 \%$ vs. $15 \%, \mathrm{P}<0.0001 ; 70 \%$ vs. $50 \%, \mathrm{P}<0.0002$, respectively). There was a significant difference in median overall survival between the two groups, being 51 months for $\mathrm{H}$ (95\% CI: 34.6-66.9) and 99 months for NHW (95\% CI: 77.3-120.7) $\mathrm{P}<0.0001$. In multivariate analysis, age (OR: 1.02, 95\% CI: 1.02-1.03, P<0.0001), poor differentiation (OR: $1.21,95 \% \mathrm{CI}$ : 1.02-1.43, $\mathrm{P}<0.02$ ), ethnicity (OR: $1.69,95 \% \mathrm{CI}: 1.07-2.55, \mathrm{P}<0.02$ ), and stage (OR: $1.95,95 \% \mathrm{CI}$ : $1.77-2.15, \mathrm{P}<0.0001$ ) were independent predictors of survival.
\end{abstract}

Conclusions $\mathrm{H}$ patients were diagnosed with gastric cancer at a younger age, to present with advanced disease at diagnosis, and had shorter overall survival compared to NHW.

Keywords Gastric cancer, Hispanic, cancer, ethnicity, survival, risks factors, health disparities, early gastric cancer

Ann Gastroenterol 2016; 29 (2): 1-7

Departments of anternal Medicine, Newark, Rutgers-New Jersey Medical School (Narjust Duma, Larysa J. Sanchez, Yulanka S. Castro); ${ }^{b}$ Medical and Surgical Oncology, John Theurer Cancer Center, Hackensack University Medical Center, Hackensack (Andrew A. Jennis, Donald A. McCain, Martin E. Gutierrez, Zubin M. Bamboat), NJ, USA

Conflict of Interest: None

Correspondence to: Narjust Duma, MD, Rutgers-New Jersey Medical School, Internal Medicine Department, 150 Bergen St., Suite UH-I248, Newark, New Jersey 07103, USA, Tel.: +1 206841 0615,

Fax: +1 973972 3129, e-mail: narjustperezmd@gmail.com

Received 21 May 2015; accepted 21 March 2016; published online 7 April 2016

DOI: http://dx.doi.org/10.20524/aog.2016.0030

\section{Introduction}

Although the incidence of gastric cancer continues to decline, it remains a common cause of cancer-related deaths in the United States and the world [1,2]. Approximately 22,220 patients are diagnosed annually in the United States [3]. While surgery remains the only curative option, 5-year survival of patients undergoing curative resection for gastric adenocarcinoma is $20-30 \%$ with a median survival of only 24 months. Despite the success of modern chemotherapy in the treatment of large bowel cancers, the 5-year survival of advanced gastric adenocarcinoma is 3.1\% [4,5].

Several studies have evaluated the association between ethnicity and survival among patients with gastric cancer in 
the past 10 years, with a great number of studies trying to find an explanation for the disparity in gastric cancer outcomes between Western and Eastern countries [6-9]. Most of the studies concluded that Asian patients have higher incidence of gastric cancer but better outcome compared with other ethnic groups, with African Americans having the worse overall survival [6-9]. More recent studies in Southern California and Texas revealed that Hispanic $(\mathrm{H})$ patients may have distinct tumor characteristics and worse outcomes when compared with individuals from other ethnic backgrounds including patients of Asian descent [10-15]. A retrospective study from the University of Texas, M.D. Anderson Cancer Center demonstrated unique features of gastric adenocarcinoma in the $\mathrm{H}$ population but ethnicity did not influence overall survival in that study [10]. Higher prevalence of predisposing risk factors including: Helicobacter pylori (H. pylori) infection, EpsteinBarr virus exposure as well as lack of preventive care services and differences in treatment were used to explain the divergent outcomes between $\mathrm{H}$ and other ethnic groups [12-15].

The existing data on $\mathrm{H}$ with gastric cancer focuses on differences in incidence and prevalence, but they lack insight into the association between ethnicity, tumor characteristics and survival. A better understanding of the factors that may explain the racial and ethnic disparities in gastric cancer outcome appears necessary to potentially improve survival for patients at risk [16-18]. With the majority of North American studies focusing on the populations of Southern California and Texas, we found it essential to conduct an analysis of the heterogeneous $\mathrm{H}$ population in Hackensack, New Jersey. The objective of our study was to compare the clinicopathologic characteristics between $\mathrm{H}$ and non-Hispanic whites (NHW) with gastric adenocarcinoma, with the goal of determining the association between ethnicity, clinicopathologic features and survival in our patient population.

\section{Patients and methods}

With approval of the Institutional Review Board and in accordance with Health Insurance Portability and Accountability Act regulations, we conducted a retrospective review of all patients diagnosed with gastric adenocarcinoma between January 1999 and March 2013 at the John Theurer Cancer Center (JTCC) at Hackensack University Medical Center. Diagnosis of gastric adenocarcinoma was made according to the 2010 World Health Organization (WHO) and the American Joint Committee on Cancer (AJCC) histologic stage classification of gastric carcinoma. In order to be included in the study the following characteristics were required: initial diagnosis of gastric adenocarcinoma had to been made in our institution or confirmed by our pathology department; age > 18 years; precisely recorded: tumor location, stage and histologic subtype; follow up at our institution after initial treatment (surgery, chemotherapy or radiation) (minimum 5 outpatient visits after the time of diagnosis); and accurately recorded vital status and time of death. Exclusion criteria comprised:history of prior malignancy or presence of metachronous tumors; patients with other gastric neoplasms, such as neuroendocrine tumors, lymphoma, or sarcoma; unknown ethnicity or racial self-identification; gastric tumors with site classified as "unspecified" or histologic type classified as "not otherwise specified"; and patients diagnosed at the time of death or by autopsy.

The patient cohort was divided into two ethnic groups, $\mathrm{H}$ and NHW. We analyzed patient demographics, tumor clinicopathologic features, and overall survival. Tumor-related features included: primary tumor location divided into cardia, fundus, body, antrum, and pylorus, clinical and pathologic stage distributed between stages I to IV (based on AJCC $7^{\text {th }}$ edition), and histologic differentiation into well, moderate, poorly differentiated and undifferentiated tumors. Treatment modalities included: chemotherapy only or radiotherapy only (no surgery); combined chemoradiation without surgery; surgical treatment only or surgical single-modality treatment (chemotherapy or radiotherapy); and surgical resection, including chemoradiation therapy and others. Recurrence rate and survival were calculated in months and analyzed.

\section{Statistical analyses}

Data analysis was performed using the SPSS statistical software (SPSS for Windows, version 19.0, SPSS, Inc., Chicago, IL). Patient characteristics and pathologic features were compared by one-way analysis for continuous variable and the chi-square test was use for categorical variables. Multinomial logistic regression analysis was used to compare clinical and pathologic characteristics among the two ethnic groups. For survival calculations, the date of diagnosis was defined as the starting point and date of last follow up with an established vital status (death vs. alive) was defined as the endpoint. Median overall survival was obtained using the Kaplan-Meier method for both ethnic groups and univariate comparison between the two groups was carried out by using the log-rank test. Cox proportional hazard method was used to examine the effect of race and ethnicity on mortality and results were presented as hazard ratios (HRs) and 95\% confidence intervals (CIs). Variables considered in the Cox model included all relevant clinical and pathologic factors. The significant level was set at 0.05 to identify the effect of possible prognostic factors on median overall survival.

\section{Results}

From 1999 to March of 2013, 807 patients were diagnosed with gastric adenocarcinoma at the JTCC, of which 638 patients met the inclusion criteria. There were $101 \mathrm{H}$ and 537 NHW with a median follow up of 24 months. The median age at diagnosis was 63 years in $\mathrm{H}$ and 69 years in NHW. A significant difference in age was found in the Stage IV subgroup, with a median age at diagnosis of 54 years for $\mathrm{H}$ and 67 years for NHW (Table 1). 
Table 1 Median age at time of diagnosis (grouped by stage), Hispanics vs. non-Hispanic whites

\begin{tabular}{lccc}
\hline & Hispanics & Non-Hispanic whites & P value \\
\hline $\mathrm{N}$ & 101 & 537 & \\
Age (all stages) & 63 & 69 & 0.05 \\
Stage I (age) & 69 & 75 & 0.07 \\
Stage II & 65 & 69 & 0.08 \\
Stage III & 60 & 68 & 0.05 \\
Stage IV & 54 & 67 & 0.04 \\
\hline
\end{tabular}

Table 2 Gastric cancer clinicopathologic characteristics by ethnicity

\begin{tabular}{|c|c|c|c|}
\hline Characteristics & Hispanics & $\begin{array}{l}\text { Non-Hispanic } \\
\text { whites }\end{array}$ & $\mathrm{P}$ value \\
\hline \multicolumn{4}{|l|}{ Stage at presentation } \\
\hline I & $9 \%(10)$ & $18 \%(97)$ & $<0.04$ \\
\hline II & $18 \%(18)$ & $15 \%(80)$ & 0.45 \\
\hline III & $25 \%(25)$ & $31 \%(166)$ & 0.21 \\
\hline IV & $48 \%(48)$ & $36 \%(194)$ & $<0.03$ \\
\hline \multicolumn{4}{|l|}{ Tumor Location } \\
\hline Cardia & $12 \%(12)$ & $43 \%(229)$ & $<0.0001$ \\
\hline Fundus & $6 \%(6)$ & $11 \%(60)$ & 0.06 \\
\hline Body & $12 \%(12)$ & $12 \%(67)$ & 0.86 \\
\hline Antrum & $36 \%(36)$ & $12 \%(66)$ & $<0.0001$ \\
\hline Pylorus & $8 \%(8)$ & $3 \%(17)$ & $<0.03$ \\
\hline Overlapping lesions & $26 \%(27)$ & $18 \%(98)$ & $<0.04$ \\
\hline \multicolumn{4}{|l|}{ Histologic grade } \\
\hline Well differentiated & $4 \%(4)$ & $11 \%(59)$ & $<0.02$ \\
\hline Moderately differentiated & $16 \%(17)$ & $34 \%(184)$ & $<0.0005$ \\
\hline Poorly differentiated & $70 \%(71)$ & $50 \%(271)$ & $<0.0002$ \\
\hline Anaplastic & $9 \%(9)$ & $4 \%(23)$ & $<0.05$ \\
\hline \multicolumn{4}{|l|}{ Histologic subtype } \\
\hline Intestinal & $34 \%(34)$ & $42 \%(226)$ & 0.24 \\
\hline Diffuse & $49 \%(50)$ & $35 \%(188)$ & $<0.04$ \\
\hline Mixed & $8 \%(8)$ & $19 \%(102)$ & $<0.02$ \\
\hline Unclassifiable & $9 \%(9)$ & $4 \%(21)$ & 0.15 \\
\hline \multicolumn{4}{|l|}{ Adjuvant therapy } \\
\hline Chemotherapy & $77 \%(78)$ & $69 \%(369)$ & 0.08 \\
\hline Radiation & $18 \%(18)$ & $29 \%$ (157) & 0.02 \\
\hline
\end{tabular}

When ethnic groups were compared by stage at presentation, $\mathrm{H}$ were more likely to present with advanced disease. Forty-eight percent of $\mathrm{H}$ had stage IV at the time of diagnosis compared with only $36 \%$ of NHW $(\mathrm{P}<0.03)$ (Table 2$)$. In addition, there was a significant difference in the stage I group with only $9 \%$ of $\mathrm{H}$ having stage I disease at the time of diagnosis vs. $18 \%$ of the NHW $(\mathrm{P}<0.04$, Table 2).
$\mathrm{H}$ were more likely to have distal tumors (antrum and pylorus) compared with NHW $44 \%$ vs. $15 \%$, $(\mathrm{P}<0.0001)$. In contrast, cardia tumors were more common in the NHW group, $43 \%$ vs. $12 \%(\mathrm{P}<0.0001)$ (Table 2$). \mathrm{H}$ were more likely to present with poorly differentiated tumors compared with the NHW, with a marked difference of $70 \%$ vs. $50 \%(\mathrm{P}<0.0002)$ (Table 2). Diffuse gastric cancer was more common in $\mathrm{H}$ patients than NHW ( $49 \%$ vs. $35 \%, \mathrm{P}<0.04$, Table 2 ).

\section{Survival}

$\mathrm{H}$ demonstrated worse survival compared to NHW, with a significant difference in median overall survival of 64.5 months for $\mathrm{H}$ (95\%CI: 45.8-83.0) and 107.6 months for NHW (95\%CI: 94.9-120.3) $(\mathrm{P}<0.0001)$ (Fig. 1). When controlling for stage, there was a significant difference in overall survival between the two ethnic groups with stage II and III disease. For stage II, $\mathrm{H}$ had a median overall survival of 58 months (95\% CI: 38.577.5) versus 104 months in the NHW (95\% CI: 76.3-131.7) $(\mathrm{P}<0.03)$ (Table 2, Fig. 2). Likewise, $\mathrm{H}$ with stage III disease had a median overall survival of 51 months (95\%CI: 11.2-90.8) versus NHW with 113 months (95\%CI: 64.0-162.0) $(\mathrm{P}<0.01)$ (Fig. 3, Table 3). Although differences in survival did not reach statistical significance for stages 1 and $4, \mathrm{H}$ tended to have lower survival at early (stage 1) and advanced (stage 4) disease (Table 3).

\section{Univariate and multivariate analyses}

Univariate and multivariate analyses were carried out to identify clinical and pathologic factors that predict median overall survival. Age, poor differentiation, stage and ethnicity were independent predictors of survival by multivariate Cox regression analysis (Table 4).

\section{Recurrence rates and disease progression}

$\mathrm{H}$ had a higher recurrence rate than NHW $18 \%(\mathrm{n}=18)$ versus $9 \%$ (48), $\mathrm{P}<0.003$. Median time to primary recurrence was 14.1 months for $\mathrm{H}$ and 22.1 months for NHW. Gastric adenocarcinoma was the primary cause of death in $66 \%(n=67)$ of $\mathrm{H}$ versus $33 \%$ (177) of NHW $(\mathrm{P}<0.0001)$. From the entire cohort, only $18 \%(n=18)$ H versus $33 \%(n=177)$ of NHW were disease-free at the time of death $(\mathrm{P}<0.007)$.

\section{Discussion}

We observed significant differences in presentation, tumor characteristics and survival in $\mathrm{H}$ and NHW with gastric adenocarcinoma. $\mathrm{H}$ patients were more likely to present with more advanced disease at a younger age and had a higher rate of distal and poorly differentiated tumors. Diffuse subtype 


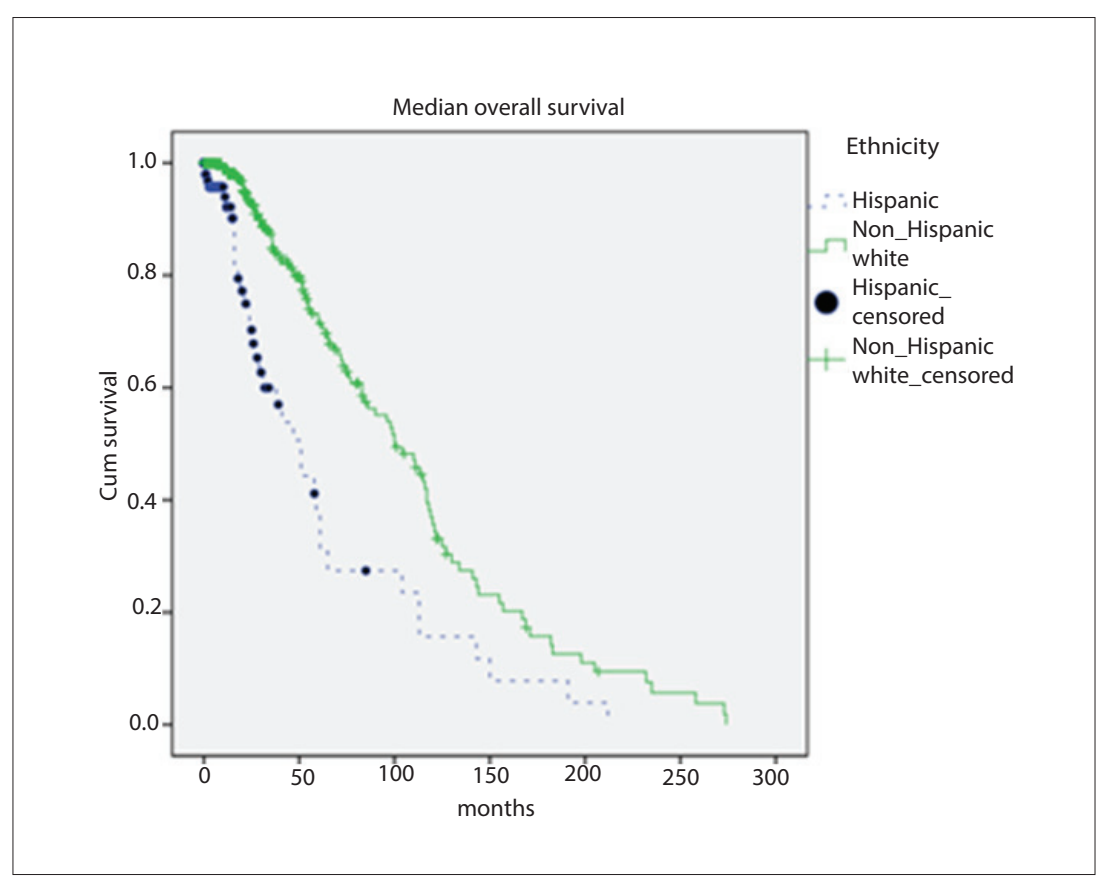

Figure 1 Kaplan-Meier for median overall survival (all stages), Hispanics vs. non-Hispanic whites

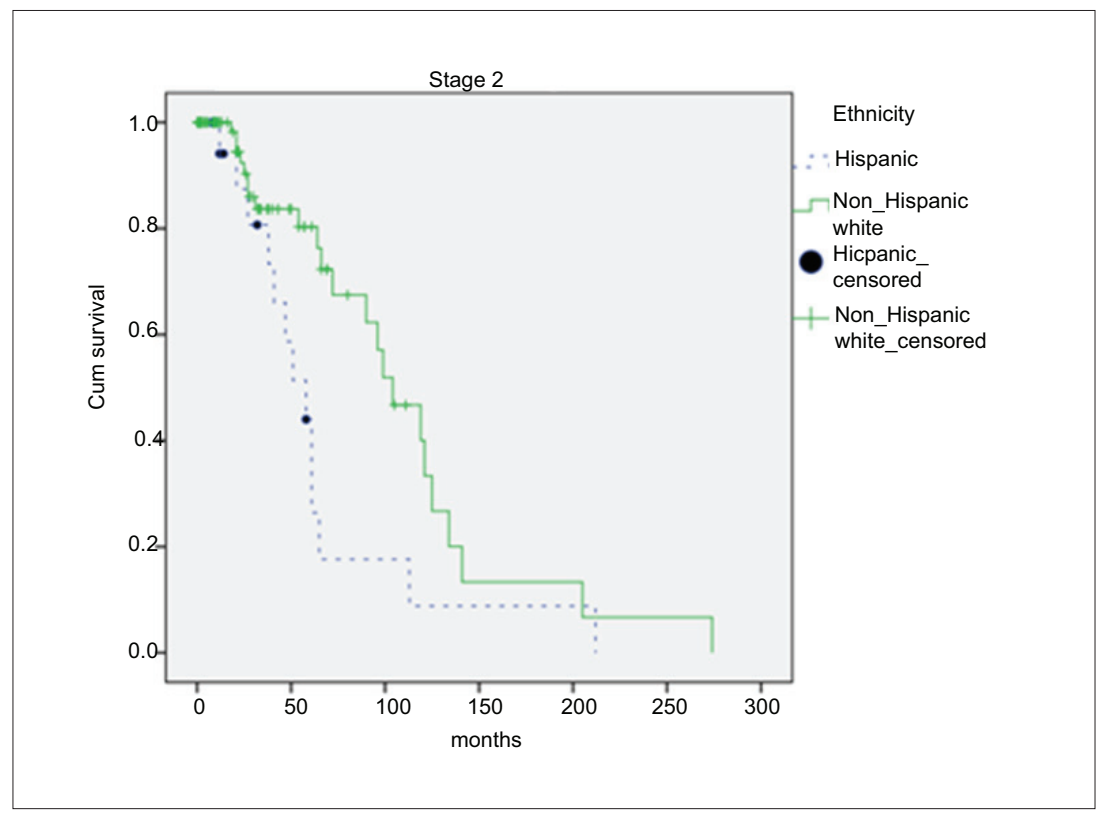

Figure 2 Kaplan-Meier for median overall survival for stage 2 gastric cancer, Hispanics vs. non-Hispanic whites

was also more prevalent in the $\mathrm{H}$ group and when comparing median overall survival, ethnicity was independent predictor of worse outcome.

In concordance with our findings, previous retrospective studies conducted in the southern parts of the United States (California and Texas) also demonstrated worse outcomes for the $\mathrm{H}$ population. For example, in 2012 Rajabi et al [15] studied 9949 patients with gastric cancer. Tumor pathobiology was found to vary by ethnicity, as NHW were more likely to have proximal tumors and less likely to have poorly differentiated or undifferentiated tumors. However, ethnicity was not studied as a predictor of survival. Likewise, in 2005 Yao et al [14] reviewed the medical records of 1,897 patients with invasive gastric cancer. They observed $\mathrm{H}$ were younger at the time of presentation when compared with other ethnic groups but in that study $\mathrm{H}$ ethnicity was not associated with gastric cancer survival when compared with other ethnic groups. In another study, Wu et al [11] who evaluated data from 37 cancer registries found that the rate of noncardia cancer was two times higher among $\mathrm{H}$ than NHW. Although our study did not evaluate some of the specific histologic patterns 


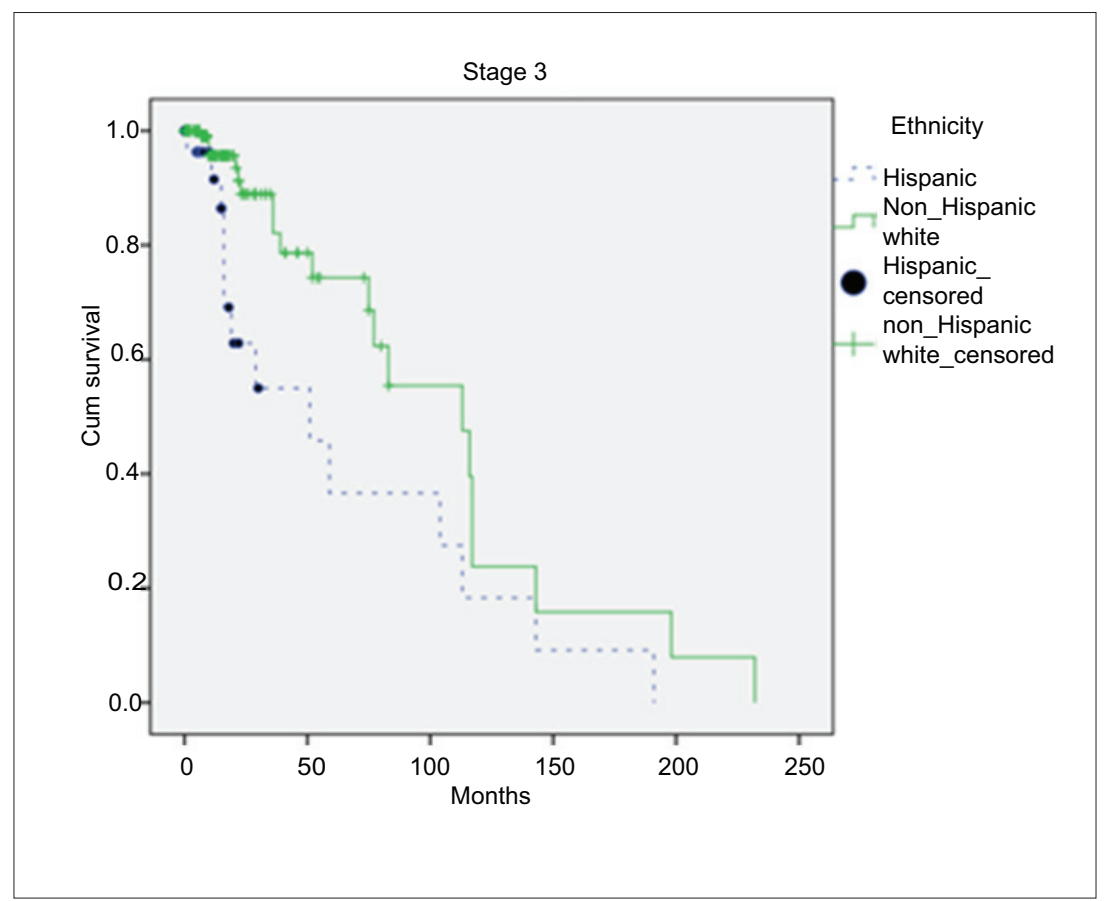

Figure 3 Kaplan-Meier for median overall survival for stage 3 gastric cancer, Hispanics vs. non-Hispanic whites

Table 3 Median overall survival (months), Hispanics vs. non-Hispanic whites

\begin{tabular}{|c|c|c|c|c|c|c|c|}
\hline & \multicolumn{6}{|c|}{$95 \%$ confidence interval } & \multirow[t]{3}{*}{$P$ value } \\
\hline & \multicolumn{3}{|c|}{ Hispanics } & \multicolumn{3}{|c|}{ Non-Hispanic whites } & \\
\hline & Estimate & Upper limit & Lower limit & Estimate & Upper limit & Lower limit & \\
\hline Stage 1 & 81.0 & 91.26 & 70.74 & 110.0 & 151.66 & 68.34 & 0.076 \\
\hline Stage 2 & 58.0 & 77.55 & 38.45 & 104.0 & 131.67 & 76.32 & $<0.03$ \\
\hline Stage 3 & 51.0 & 90.83 & 11.17 & 113.0 & 162.00 & 64.0 & $<0.01$ \\
\hline Stage 4 & 29.3 & 37.41 & 21.26 & 58.0 & 109.51 & 36.69 & $<0.06$ \\
\hline
\end{tabular}

Table 4 Predictors of survival in our gastric cancer patients (univariate and multivariate analysis)

\begin{tabular}{lcccccc}
\hline & \multicolumn{3}{c}{ Univariate } & & \multicolumn{2}{c}{ Multivariate } \\
\cline { 2 - 7 } & P value & Hazard ratio & CI: $95 \%$ & P value & Hazard ratio & CI: 95\% \\
\hline Age & $<0.0001$ & 1.013 & $1.01-1.02$ & $<0.0001$ & 1.028 & $1.02-1.03$ \\
Poor differentiation & 0.0004 & 1.716 & $1.59-1.86$ & 0.025 & 1.213 & $1.02-1.43$ \\
Anatomic location & 0.067 & 0.907 & $0.80-1.01$ & 0.074 & 0.901 & $0.80-1.01$ \\
Ethnicity & 0.0003 & 2.08 & $1.40-3.09$ & 0.02 & 1.659 & $1.07-2.55$ \\
Stage & $<0.0001$ & 1.808 & $1.64-1.98$ & $<0.0001$ & 1.057 & $1.77-2.15$ \\
Adjuvant radiation & 0.275 & 1.253 & $0.85-1.85$ & 0.803 & $0.65-1.75$ \\
Adjuvant chemotherapy & 0.007 & 0.895 & $0.74-0.95$ & 0.07 & 0.892 & $0.79-1.01$ \\
\hline
\end{tabular}

CI, Confidence interval

studied by Yao et al, we observed similar tumor characteristics. Our $\mathrm{H}$ population was more likely to present with distal and poorly differentiated or anaplastic tumors compared with NHW.
This could be partially explained by the higher incidence of $H$. pylori infection in the $\mathrm{H}$ population. Everhart et al [19] reported that $H$. pylori infection prevalence is three times higher in $\mathrm{H}$ than 
in NHW $[12,20]$. Besides socio-economic factors, there is a new theory of hereditary susceptibility to $H$. pylori infection. Studies suggest that members of certain ethnic groups including $\mathrm{H}$ have a higher rate of infection than Caucasians even after socioeconomic differences are controlled [21]. Malaty et al [22] found a strong association between variants at the Toll-like receptor 10, 1, and 6 (TLR10/1/6) locus on chromosome 4 and H. pylori serologic status, which may contribute to the understanding of host susceptibility to a common infectious disease. This theory is still being investigated but it could explain the higher incidence of $H$. pylori infection in some ethnic groups. H. pylori infection has been linked to increased risk of gastric cancer and has a direct relationship to non-proximal gastric cancer, partially explaining the higher prevalence of these tumor characteristics in our $\mathrm{H}$ patients.

In accordance with several studies, we observed that $\mathrm{H}$ patients with gastric adenocarcinoma had a higher recurrence rate and were more likely to die of gastric adenocarcinoma. Some researchers have suggested the importance of some socio-economic factors [23]. It is important to note that $\mathrm{H}$ have one of the highest uninsured rates among ethnic groups in the United States and are less likely to receive preventive health care compared with NHW [24]. This disparity in healthcare access suggests that $\mathrm{H}$ would be more likely to have delays in diagnosis and to present with more advanced disease. Our study follows this trend with almost half of the $\mathrm{H}$ patients presenting with stage IV disease when compared with $36 \%$ of the NHW.

In contrast to prior publications, we observed ethnicity to be an independent factor predicting survival. Overall our $\mathrm{H}$ patients' median survival was approx. 40 months less than NHW. We also observed a significant difference in survival after controlling for stage especially with our stage II and III patients. Receipt of chemotherapy and radiation, however, were not independent predictors of survival. Unmeasured factors, such as tumor biology and genetic mutations, have been proposed as important factors that could explain the racial differences in numerous malignancies, including gastric cancer, and could account for the observed differences in survival between the two ethnic groups in our study. The higher incidence of distal and diffuse-type tumors in $\mathrm{H}$ could support this theory $[25,26]$. Existing data suggests that different pathophysiologic pathways lead to the development of the diffuse and intestinal subtypes of gastric cancer. Defects in the cellular adhesion system are a characteristic of diffuse gastric cancer. The cadherins and catenins play an important role in these derangements. Determining whether $\mathrm{H}$ have a higher rate of cadherin deletions or inactivating mutations would be an interesting follow-up study.

The current study is unique, because it includes a diverse sample of patients from the New Jersey area. In the 2010 census data on race and ethnicity, New Jersey was named the state with the nation's seventh largest $\mathrm{H}$ population. When compared with the population in the southern United States, New Jersey has a more balanced $\mathrm{H}$ population distribution (Caribbean 34\%, Central and South American habitants 29\% and $37 \%$, respectively). In contrast, the $\mathrm{H}$ distribution in the Southern United States is skewed with 62\% Central American (including Mexican, Guatemalan and Salvadorans), 23\%

\section{Summary Box}

\section{What is already known:}

- Gastric cancer has unique socioeconomic and geographic characteristics

- Hispanic $(\mathrm{H})$ patients are more likely to have distal and poorly differentiated tumors

- Ethnic minorities with gastric cancer tend to have worse prognosis compared with Caucasians

\section{What the new findings are:}

- H were more likely to present at younger age and with more advanced gastric adenocarcinomas than non-Hispanic whites

- After accounting for age, histologic grade and stage, $\mathrm{H}$ ethnicity was a significant and independent predictor of survival

South American and 15\% Caribbean [27,28]. This allows us to generate conclusions that could apply to a broader $\mathrm{H}$ patient population.

The limitations of this study are: 1) its retrospective design; 2) the relatively small sample size for $\mathrm{H}$; 3) lack of data on environmental factors associated with ethnicity; and 4) inability to measure differences in tumor biology between ethnic groups by stage.

In summary, $\mathrm{H}$ were more likely to present at younger age and with more advanced gastric adenocarcinomas than NHW. The higher rate of diffuse subtype and poorly differentiated tumors may explain some of the survival differences. After accounting for age, histologic grade and stage; ethnicity was a significant predictor of survival in our patient population. An ethnicity-based analysis of gastric cancer, such our study, acknowledges the intersection of biology, socioeconomic factors and access to health care with the natural history of the disease. Further research should aim to elucidate the basis of these differences, as this could alter management and improve survival.

\section{Acknowledgments}

The authors thank Mrs. Theresa Amatucci and the Hackensack University Medical Center's tumor registry staff for their help with data collection.

\section{References}

1. Jemal A, Bray F, Center MM, et al. Global cancer statistics. Cancer J Clin 2011;61:69-90. 
2. Siegel R, Naishadham D, Jemal A. Cancer statistics for Hispanics/ Latinos. Cancer J Clin 2012;62:283-298.

3. Brenner H, Rothenbacher D, Arndt V. Epidemiology of stomach cancer. Methods Mol Biol 2009;1:467-477.

4. Karpeh M, Kelsen D, Tepper J. Cancer of the stomach. In: DeVita V, Hellman S, Rosenberg S editors. Cancer Principles \& Practice of Oncology. Volume $1,6^{\text {th }}$ edition, Lippincot, Williams \& Wilkins; Philadelphia, 2001:1092-126.

5. Theuer CP. Asian gastric cancer patients at a southern California comprehensive cancer center are diagnosed with less advanced disease and have superior stage-stratified survival. Am Surgeon 2000;66:821-826.

6. Al-Refaie M, Waddah B, Jennifer F, et al. The impact of ethnicity on the presentation and prognosis of patients with gastric adenocarcinoma. Cancer 2008;113:461-469.

7. Kim J, Sun CL, Mailey B, et al. Race and ethnicity correlate with survival in patients with gastric adenocarcinoma. Ann Oncol 2010;21:152-160.

8. Gill S, Shah A, Le N, et al. Asian ethnicity related differences in gastric cancer presentation and outcome among patients treated at a Canadian cancer center. J Clin Oncol 2003;21:2070-2076.

9. Kubo A, Corley DA. Marked multi-ethnic variation of esophageal and gastric cardia carcinomas within the United States. Am J Gastroenterol 2004;99:582-588.

10. Wu X, Vivien W, Chen P, et al. Incidence of esophageal and gastric cancers among Hispanics, non-Hispanic whites and non-Hispanic blacks in the United States: subsite and histology differences. Clin Cancer Res 2007;18:585-593.

11. Wiggins CL, Becker TM, Key CR, Samet JM. Stomach cancer among New Mexico's American Indians, Hispanic whites, and non-Hispanic whites. Cancer Res 1989;49:1595-1599.

12. Yao JC, Schnirer II, Reddy S, et al. Effects of sex and racial/ethnic group on the pattern of gastric cancer localization. J Gastric Cancer 2002;5:208-212.

13. Yao JC, Tseng JF, Worah S, et al. Clinicopathologic behavior of gastric adenocarcinoma in Hispanic patients: analysis of a single institution's experience over 15 years. J Clin Oncol 2005;23:3094-3103.

14. Rajabi B, Corral JC, Hakim N, Mulla ZD. Descriptive epidemiology of gastric adenocarcinoma in the State of Texas by ethnicity: Hispanic versus White non-Hispanic. J Gastric Cancer 2012;15:405-413.

15. Jinjuvadia R, Jinjuvadia K, Liangpunsakul S. Racial disparities in gastrointestinal cancers related mortality in the US population. Dig Dis Sci 2012;58:236-243.

16. Laiyemo AO, Doubeni C, Pinsky PF, et al. Race and colorectal cancer disparities: health-care utilization vs different cancer susceptibilities. J Natl Cancer Inst 2010;102:538-546.

17. Steyerberg EW, Earle CC, Neville BA, Weeks JC. Racial differences in surgical evaluation, treatment, and outcome of locoregional esophageal cancer: a population-based analysis of elderly patients. J Clin Oncol 2005;23:510-517.

18. Everhart JE, Kruszon-Moran D, Perez-Perez GI, Tralka TS, McQuillan G. Seroprevalence and ethnic differences in Helicobacter pylori infection among adults in the United States. $J$ Infect Dis 2000;181:1359-1363.

19. Chow WH, Blaser MJ, Blot WJ, et al. An inverse relation between cag-A strains of Helicobacter pylori infection and risk of esophageal and gastric cardia adenocarcinoma. Cancer Res 1998;58:588-590.

20. Graham DY, Malaty HM, Evans DG, et al. Epidemiology of Helicobacter pylori in an asymptomatic population in the United States. Effect of age, race, and socioeconomic status. J Gastroenterol 1991;100:1495-1505.

21. Malaty HM, Engstrand L, Pedersen NL, Graham DY. Helicobacter pylori infection: genetic and environmental influences-a study of twins. Ann Intern Med 1994;120:982-986.

22. Gammon MD, Schoenberg JB, Ahsan H, et al. Tobacco, alcohol, and socioeconomic status and adenocarcinomas of the esophagus and gastric cardia. J Natl Cancer Inst 1997;89:1277-1284.

23. Derose K, Escarce J, Lurie N. Immigrants and health care: sources of vulnerability. Health Affairs 2007;26:1258-1268.

24. Wu MS, Shun CT, Wang HP, et al. Genetic alterations in gastric cancer: relation to histological subtypes, tumor stage, and Helicobacter pylori infection. Gastroenterology 1997;112:1457-1465.

25. Ottini L, Palli D, Falchetti M, et al. Microsatellite instability in gastric cancer is associated with tumor location and family history in a high risk population from Tuscany. Cancer Res 1997;57:4523-4529.

26. Cooper RS, Kaufman JS, Ward R. Race and genomics. N Engl J Med 2003;348:1166-1170.

27. US Census Bureau. Profile of general population and housing characteristics: 2010 demographic profile data. American Factfinder 2010;10:1-5.

28. 2006-2008 American Community Survey 3-Year Estimates for Hackensack city, New Jersey, United States Census Bureau. Accessed July 20 2014. 